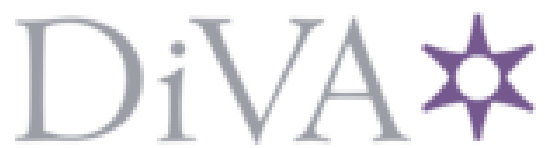

http://www.diva-portal.org

This is the published version of a paper presented at 28th European Safety and Reliability Conference, ESREL 2018 in Trondheim, Norway, June 17-21, 2018.

Citation for the original published paper:

van Laere, J., Berggren, P., Ibrahim, O., Larsson, A., Kallin, S. (2018)

A simulation-game to explore collective critical infrastructure resilience

In: S. Haugen, A. Barros, C. van Gulijk, T. Kongsvik, J. Vinnem (ed.), Safety and

Reliability - Safe Societies in a Changing World: Proceedings of ESREL 2018, June

17-21, 2018, Trondheim, Norway, 688 (pp. 1305-1312). London: CRC Press

N.B. When citing this work, cite the original published paper.

Permanent link to this version:

http://urn.kb.se/resolve?urn=urn:nbn:se:his:diva-15707 


\title{
A simulation-game to explore collective critical infrastructure resilience
}

\author{
Joeri van Laere \\ University of Skövde, Skövde, Sweden \\ Peter Berggren \\ Linköping University, Linköping, Sweden \\ Osama Ibrahim \\ Stockholm University, Kista, Sweden \\ Aron Larsson \\ Mid Sweden University, Sundsvall, Sweden \\ Susanne Kallin \\ Combitech AB, Sundbyberg, Sweden
}

\begin{abstract}
Resilience of interdependent infrastructures increasingly depends on collaborative responses from actors with diverse backgrounds that may not be familiar with cascade effects into areas beyond their own sector. A simulation-game can enable societal actors to obtain a deeper understanding of the interdependencies between their infrastructures and their respective crisis responses. Following a design science approach, a simulation-game has been developed that combines role-playing simulation and computer simulation. The simulation-game challenges participants to address the interaction between payment disruptions, food and fuel supply, security problems (riots, robberies) and communication challenges (preventing hoarding). A number of crucial design choices were handled while developing the simulation-game. The main design challenges were: How to validate an unthinkable escalation scenario?; How to give the simulation a sufficient level of detail on all aspects and keep the complexity graspable so it can be played instantly?; and How much time should each playing round take?
\end{abstract}

\section{INTRODUCTION}

Resilience of critical infrastructures is a complex problem area. When societal actors with different backgrounds quickly need to orchestrate a collective crisis response, a deep understanding for the existing interdependencies between their respective infrastructures and crisis response strategies is required. Gaming-simulation can help to collaboratively develop a deeper understanding of such interdependencies and be a safe environment to explore the robustness of response strategies from a multi-sectorial perspective. In the context of tightly interrelated infrastructures a response strategy should not only be beneficial for individual organizations or sectors, but even mitigate consequences and limit escalations from a holistic multi-sectorial perspective.

Building a simulation game involves many design choices. Depending on which choices are made, consciously or unconsciously, very different simulations or simulation-games can be created for studying the same problem. It is important to build simulation- games of good quality and to understand how crucial design choices impact simulation-game design and simulation-game outcomes. Consequently, the contribution of this paper is a detailed description of our simulation-game design. Following a design science research approach, a simulation-game has been created that enables actors from a large variety of critical infrastructures to analyze and mitigate the cascading effects of payment disruptions on their respective infrastructures. Besides a presentation and motivation of the most important simulation-game design choices (Section 4), three major design challenges were identified: scenario validation, game complexity and length and number of playing rounds (Section 5).

\section{THEORY BACKGROUND}

Our research builds upon three research areas: critical infrastructures, resilience and gamingsimulation. 


\subsection{Critical infrastructures and cascading effects}

Societies rely on well-functioning critical infrastructures such as Energy, Information and Communication Technology, Water Supply, Food and Agriculture, Healthcare, Financial Systems, Transportation Systems, Public Order and Safety, Chemical Industry, Nuclear Industry, Commerce, Critical Manufacturing, and so on (Alcaraz \& Zeadally 2015). When one or more critical infrastructures break down or provide only limited service, large numbers of citizens, companies or government agencies can be severely affected (Boin \& McConnell 2007, Van Eeten et al. 2011). Breakdowns can be caused by internal factors (human or technical failure), external factors (nature catastrophes, terror attacks) or by failures of other infrastructures as there are many dependencies between critical infrastructures (Van Eeten et al. 2011). Energy and Information Technology or Telecommunications are well-known event-originating infrastructures that generate cascading effects in many other infrastructures, as has been shown in different types of analyses (Van Eeten et al. 2011, Laugé et al. 2015). In times of increasing digitalisation and an ever increasing development towards a digitally interconnected society, security experts argue for more awareness for digital vulnerabilities, more attention for cyber security and a need to educate professionals and citizens on these matters (Hagen 2016).

Ansell et al. (2010) argue that resilience of interdependent infrastructures increasingly depends on collaborative responses from actors with diverse backgrounds that may not be familiar with cascade effects into areas beyond and outside their own organisation or sector. Boin \& McConnell (2007) and Van Eeten et al. (2011) argue that there is limited empirical evidence of cascading effects across many infrastructures, which makes it hard to foresee which interactions may occur across sectors. Risk analysis, business continuity management and crisis management training are often performed within the context of a single organisation or sector and are seldom addressing the holistic analysis of multiple infrastructures (Van Eeten et al. 2011).

More research is needed to understand collective resilience in the context of critical infrastructure management. In this study, a contribution is made by focusing on one application area, i.e. how payment disruptions impact other critical infrastructures. Despite the long term efforts of public and private actors in the financial sector in Sweden to identify, analyse and understand risks and to develop routines for preventing and mitigating serious disruptions in the payment system in Sweden, there is still a lack of insight into how the proposed action plans exactly need to be executed and how numerous other actors in society (e.g. citizens, food stores, gas stations, voluntary organizations, governmental agencies and so on) will act in case of a temporary or complete breakdown of the payment system. For instance, several key actors in the payment system have in earlier studies expressed that they will take a larger responsibility than their formal responsibility (MSB-2009-3309 2010), but it is not clear what this implies and how these organizations actually will act when crisis hits.

\subsection{Resilience}

Lundberg \& Johansson (2015) and Bergström et al. (2015) list that resilience amongst others can refer to: bouncing back to a previous state, or bouncing forward to a new state, or both; absorbing variety and preserve functioning, or recovering from damage, or both; and being proactive and anticipating, or being reactive (when recovering during and after events), or both. Given the variety of interpretations of resilience, resilience is hard to operationalize into measurable indicators (Lundberg \& Johansson 2015).

Lundberg \& Johansson (2015) made an effort to merge and compile different points of view in the field of disaster and crisis response resilience into one systemic model, the Systemic Resilience Model (SyRes). The model departs from the idea that the coping with an unwanted event can be seen as a downward spiral activating certain basic resilience functions (anticipation, monitoring, responding, recovery and learning) and their associated strategies (where the strategies are the actual manifestation of the functions, or their 'form', which may differ from system to system). Further, Lundberg \& Johansson (2015) suggest that resilience is needed to protect core values, i.e. values central for the existence of the system in focus. In safety-critical systems, such core values usually take the form of maintaining safety, such as avoiding harm to humans or critical infrastructures. For a commercial business such as a grocery store, a petrol station or a bank, a core value is typical to create revenue, i.e. to assure a higher income than outcome. Without this profit, the business will seize to exist. This core value will manifest itself in a number of practical activities which usually take the form of different flows such as goods, money, services etc.

In line with the challenges to resilience suggested by Johansson \& Lundberg (2010) comes the fact that most systems in society, such as the payment system, depend on several different actors to function properly. Therefore, resilience must be considered from a systems perspective. In the field of resilience, this is sometimes referred to as 'collective resilience'. Weick \& Sutcliffe (2007) argue that 
loosely coupled systems relying on a 'sensemaking' process generally are more resilient than tightly coupled systems based on the assumption that all system states can be predicted and safeguarded against possible threats. This resembles distinctions made in safety science between the paradigms labelled Safety I and Safety II (Hollnagel 2013) where Safety I is signified by the idea that safety can be designed into a system and Safety II is signified by the idea that human adaptability is the most important contributor to success despite inadequate design or insufficient predictive capacity of safety engineers. Weick \& Sutcliffe (2007) argue that a dilemma exists in sensemaking: you can optimise for analysis or action, but not both. This dilemma seems contradictory to the requirements of resilience, because Weick \& Sutcliffe argue for sensitivity to operations and reluctance to simplify (i.e. an interest in details and scrutinize the situation at hand) and simultaneous blunt and immediate action without thorough analysis. The solution suggested by Weick \& Sutcliffe (2007) is that deep knowledge about the system should have been acquired earlier (long before the disruption) so that quick and blunt action based on deep understanding of the system's dynamics is possible in case of disruptions. As more actors may simultaneously initiate a quick and blunt response, a risk is that these responses counteract each other. Weick \& Roberts (1993) discuss how attentiveness (heedful interrelating) is key in a resilient group response, i.e. while acting quick and blunt, various actors should pay close attention to how other actors respond and to what kind of system behaviour their collective response leads. Heedful interrelating has been demonstrated in small groups. Heedful interrelating becomes challenging when systems become larger, more interrelated and involve more and more decision makers that do not really know each other and do not understand the impact of their decisions on nearby systems, as in the case of large interdependent infrastructure systems (Ansell et al. 2010). Then these groups of stakeholders may lack swift trust (Weick \& Roberts 1993) and may lack a shared understanding of the situation and a shared vision, which may lead to inferior performance (Berggren et al. 2014). Yet another risk might be organisations or companies who continue putting their own goals ahead of the common good, thus risking initiating counterproductive actions that may hamper the process of recovery from disruptions.

\subsection{Gaming-simulation}

Gaming-simulation is defined as a specific form of simulation. Simulation in general aims at designing a model of a system in a complex problem area in other to be able to experiment with the model. Deeper insight in the behavior of the system is created by evaluating various operating strategies against each other in one ore multiple scenarios. Gaming-simulation differs from other forms of simulation in that it incorporates roles to be played by participants and game administrators, implying that people and their (goal-directed) interactions become part of the simulation (Laere et al. 2006). In addition to role descriptions and interaction formats, simulation-games can also include a physical simulation model (a board game, a mock-up, a computer simulation, or any other representation of a physical reality) which the game participants need to interact with. It is important to understand that both the changes and impacts of changes to the physical simulation model in the simulationgame and the interaction between the participants (often negotiation processes about what to change and how to interpret changes in the physical simulation model) are part of the simulation-game and object of study (Mayer 2009). Gaming-simulation is especially relevant when the "how and why" of the interaction processes between the participants are of interest and when these interactions cannot easily be incorporated in computer simulation models. In addition, it creates a deeper learning opportunity, as simulation-game participants literally are active participants in the simulation, rather than passive observers of a computer simulation.

To design a high quality simulation-game, many design choices have to be taken into account, which often are not self-evident, but rather involve tricky cost-benefit analyses ending up with a dilemma (is the benefit worth the extra cost?). Examples of such design choices are for example (Laere 2003, Mayer 2009, Meijer 2009): defining a limited number of research or learning objectives, defining the number and content of roles, defining the scope of the modelled situation/problem, guaranteeing the validity of the simulation, defining rules and constraints, defining the load (difficulty), choosing the location/ environment where the game will be played, selecting the type of participants to be invited, design of qualitative and quantitative data collection during the game, degree of realism of the scenario, degree of complexity of the game (often phrased as modelling internal complexity of the system to be modelled, but creating external simplicity, i.e. an easy to understand and easy to play game for the participants), degree of competition, degree of dynamics, macro cycle (preparation, playing, debriefing, follow-up), micro-cycle (number of playing rounds) and real-time or symbolic-time.

\section{RESEARCH DESIGN}

Our research design is based on an inductive research strategy and a qualitative research method. 
A clear theory on how critical infrastructures exactly are related, and how the many actors involved collaboratively could manage disruptions that create cascading effects in many infrastructures, is lacking. As such, there is a need for theory building rather than theory testing, which leads us to an inductive research strategy (Eisenhardt \& Graebner 2007). From an interpretative perspective, we are interested in exploring the many different interpretations of actors involved regarding what challenges disruptions can pose and how they could be handled collaboratively across the affected infrastructures. A simulation-game can be a safe environment where participating actors can experiment with different action alternatives, and through their participation and their choice of resilience strategies demonstrate the core values they hold.

For the design of the simulation-game a design science research strategy is adopted. The result of design science research is a purposeful artifact created to address an important organizational problem (Hevner et al. 2004). In our case, the problem is "understanding critical infrastructure dependencies and exploring collective infrastructure resilience strategies" and the artifact is "a simulation-game that can serve as save analysis, learning and exploration environment". As argued in Hevner et al. (2004) design science is an iterative search method aiming at identifying a creative solution for the problem at hand. Given our interpretative stance, our aim is not to design the best or an optimal simulation-game, but rather to design one appropriate simulation-game (amongst many alternatives), and developing a deep understanding what the benefits and drawbacks of our chosen design are. Design science addresses relevance by a strong interest the societal needs in the application environment studies, and aims simultaneously at rigor through reflecting on the design process and arguing how the produced solution informs the research front (where either the produced artifact and/or the insights regarding how to design such an artifact can be research contributions).

A first data collection phase consisted of document study of prior incidents (33 reports), 6 interviews with key representatives from each sector and two half-day workshops with respectively 26 national and 11 local actors in order to identify cascading effects, consequences, actors involved and potential mitigating actions which they could perform with regard to payment disruptions (Laere et al. 2017a). Mapping these characteristics of our problem environment contributed to identification of the elements to be simulated in our simulationgame. A second data collection phase aimed at analysing existing simulation-games for critical infrastructure resilience (Laere et al. 2017b). Here, six existing simulation-games where analysed in detail with the purpose of understanding how different design choices impact the capabilities of the learning environment and the learning experience of the participants.

Next, the collected data was analysed and transformed to elements of the envisioned simulationgame. During a series of six bi-monthly organised full day workshops with the project team of 10 researchers, different versions of the simulationgame were created, tested and refined. In between the workshops the involved researchers worked in smaller task forces on different elements of the simulation-game. During the last to full day workshops societal actors from the different sectors were involved to gather their feedback on the simulation-game design. The next two sessions summarize the main design choices and main design challenges that were identified and dealt with under this design process.

\section{GAME DESIGN CHOICES}

\subsection{Game overall structure}

When role playing simulation games and computer simulations are combined a powerful simulation environment is created. Actors, as game participants, can collaborate or compete with each other in different rounds, enter their decisions in the computer simulation and receive the output of the computer simulation as input in their next playing round. As such, participants can experience social interaction (role playing) and large scale system dynamics (impacts of their decisions over time, or on a large scale). The participating decision makers can compare intended consequences with unintended and unexpected consequences and create a deeper understanding of the system as a whole and the behavior of other game participants.

The main purpose of the simulation-game is to create a deeper understanding of the dynamics and interdependencies in the overall system. Alternatively or additionally, collaboration between the different actors involved could be a learning goal. When collaboration is a learning goal, actors may be placed in different rooms and different actors may have different information at hand. In such games sharing the right information with the right actor at the right time might be in focus. In our design became clear quite early that grasping the complexity of the overall societal system (i.e. all sectors that are impacted by payment disruptions) and their interactions is a challenge at such. It was decided that grasping this complexity created sufficient load and that additional collaboration challenges would adventure the main objective of understanding overall system dynamics. Therefore 
it was decided that the players, who each can represent different societal roles (i.e. food sector, fuel sector, media etc.) would be placed in one team that in collaboration would try to manage payment disruptions.

Putting the participants in one team makes the use of simulation-game flexible. Teams could consist of either 3, 5, 7, 9 or 11 participants interacting as one team with the computer simulation. From a learning perspective it is preferable to have a larger group with a strong diversity in backgrounds, but from an execution perspective it is a benefit that a simulation-game session still can be performed even if two of the seven participants would not show up.

The team interacts with a fictive society represented in the computer simulation. The computer simulation is created with Anylogic simulation software. The main reason to choose this software package is that it enables to combine agent-based simulation, discrete event simulation and system dynamics simulation, which gives us a certain flexibility to implement different scenarios. The computer simulation covers a typical region with some cities and some countryside, where relevant societal infrastructures can be distinguished (see 4.2). The overall idea is that payment disruptions occur (see 4.3) in this fictive society and that the team can try out different combinations of actions strategies (see 4.4) to learn how they differ in impact on a number of performance criteria (see 4.5). An important characteristic of the simulation-game is that the participating teams can re-play the same scenario over and over again (see 4.6). By keeping the scenario conditions constant the participants can really compare their chosen action strategies and experience and learn how different combinations of actions give different impacts.

During the design process we have alternated between versions that could be played at a distance, or at one physical location. Playing at a distance allows for more elaboration time between playing rounds which might be beneficial for learning (i.e. making more thoughtful choices). While keeping the alternative of playing at a distance as a potential future development, our current impression is that the intense discussion and interaction between the participants in the team are of major importance (as the learning and creation of deeper insight occurs exactly there). Therefore physical presence at one location is to be preferred.

\subsection{Sectors represented in the computer simulation}

From the document studies and workshops with societal actors (Laere et al. 2017a) a number of societal actors, sectors and processes has been selected that are primarily vulnerable for payment disruptions and therefore form the core of computer simulation of the fictive society in the simulation-game.

The fictive society consists of a number of grocery stores of varying size, a number of fuel stations and a number of pharmacies (where medicine can be bought). For each store a customer flow is created. The number of customers, their demands, and the number of stores are balanced based on statistics for typical regions in Sweden. Stores offer one or several of the following payment options (card payment, cash payment, digital phone payments and delayed invoice payments). Individual customers have also one or more different payment options available. When customers collect goods in the store the store's payment options and their payment preferences need to match to create a transaction. Payment transactions are performed and accredited by the actors from the finance sectors (i.e. credit card companies and/or banks) and lead to account changes for stores and customers. When goods are sold new goods are order and delivered by transport companies. Customers and transport companies consume fuel, which in turn requires financial transactions when they buy new fuel. ATMs are available for those customers and transport companies who want to acquire cash and ATMs are refilled by certain transport companies. Security guards are present at the larger stores, and more could be hired when needed. Different media actors are represented who can spread news which in turn can influence consuming behavior.

In our current implementation there is a rather rough logic. The purpose in the development has been to quickly arrive at an implementation that can be played with actual representatives from different critical infrastructure managers. Given their feedback in early playing sessions the simulationgame will be further refined. Our aim is to perform 30 playing sessions in 2018 and 2019 and gradually improve the design science artifact under study.

\subsection{Payment disruption scenario}

Thus far one main scenario has been developed and implemented. During the course of our project (2016-2021) two additional scenarios will be created. Our current scenario is a 10-day card payment disruption at the store level. The other scenarios will be developed in such a way that they effect other parts of the payment system (i.e. disruptions in the transferring of money between accounts - or a long term scenario that covers multiple years rather than only a few days).

The current 10 day card-payment scenario is based on the fact that $90 \%$ of transactions in stores in Sweden is based on card payment, which makes 
the Swedish society extremely dependent on that payment option as the other alternatives are not capable to instantly handle such large volumes of transactions. Although the scenario is much more detailed than presented here, the main elements of the scenario are as follows.

Day 1: Card payment disappears as payment option. The expectation of most actors is that it will take some hours. Stores close or offer digital phone payments or cash payments as alternatives. Chaotic scenes for those customers who are disappointed. Queues at stores and at ATMs.

Day 2-3: Banks and media announce that the disruption will take several days. Customers are confused where they can buy. Sales drop dramatically, use of cash and digital payments increase dramatically, some customers start hoarding, deliveries and logistics to stores are a mess as major fluctuations occur. A lot of cash in stores and in society at large increase robbery risks.

Day 4-5: Cash and digital payment options collapse as well as they cannot cope with the large volumes. Long queues, angry customers as they are running out of goods at home, customers become aggressive, a lot of stores close, those who are open experience massive hoarding. Perishable goods need to be thrown away as they cannot be sold. Logistics trouble increases.

Day 6-7: Government in collaboration with stores introduce a general "buy based on your identity and pay later by invoice option". Massive hoarding when stores open. Logistics collapse again as they have hard to adjust from total sales stop to massive hoarding.

Day 8-10: The general "buy based on your identity and pay later by invoice option" is too complicated and time consuming which creates enormous queues, frustration and aggression. Chaos and panic on more and more places. Police and army guard the few stores that still keep open.

The cascading effects that occur are not hard implementations, but do occur as cascading effects as a result of the initial card payment disruption. All other effects can be influenced when other actions are chosen by the players.

\subsection{Action alternatives to mitigate disruptions}

The team that plays the simulation-game in several rounds can select on ore more of the following actions. Besides these alternatives that are given (and prepared) we are open for creative ideas of the participants. When they come with a suggestion for an unforeseen action the game facilitators will try to simulate that action and its presumed impacts instantly in the simulation if possible.
Possible actions that the team can select are for example (note that each action can be implemented at any day in the scenario): offer more/less payment options at all or some stores; close or open stores; increase/decrease deliveries to stores; communicate information or instructions to customers; offer cash withdrawal in stores; limiting the amount of goods per purchase; increase/decrease the number of security guards for one or several stores; throw away perishable goods; give away perishable goods for free.

The design of the computer simulation involves an implementation of impacts of each and every action, based on interviews and discussions with key representatives from the different societal processes simulated. Even as we as designers know the approximate impact of individual action, the playing sessions need to reveal how the different actions in combination fall out. In addition, actions can be implemented on different moments in time (day one to ten in the scenario), which makes the number of alternative strategies near to infinite. Rather than experimenting with the computer simulation as such ourselves, the whole idea with involving real societal actors in role-playing is to let their expertise and value frames guide the selection and time-planning of combinations of actions. Moreover, not only the selection of actions as such is of interest, but also the motivation and reasoning behind. Therefore, the teams who play need to motivate the timing and selection of actions before they are implemented in various playing rounds and the collection of these motivations is seen as a crucial element of the simulation-game.

\subsection{Performance metrics}

Extensive discussions have been held at several of our design workshops and in intermediate work group meetings considering what indicators are most relevant and appropriate to visualize performance in the various sectors of society. Currently, three major performance areas have arisen: 1) payment options, 2) good flows, and 3) security

Available payment options are statistics on the actual use of each of the four different payment over time, or the amount of stores (in $\%$ of total stores) where they each option is available.

For good flows the main indicators is "disappointed customers" over time (the simulation counts the number of arriving customers that cannot fulfil their purchase for any reason). Additionally it is shown how many stores currently are closed (in \%), which groups of goods currently are out of stock, how many perishable goods are destroyed over time, and how many planned deliveries that fail (due to fuel shortages). 
Security related indicators are amount of cash in stores (implying increased robbery risk), number of shop lifting incidents, and the number of security guards per store.

A performance area which has been suggested but been hard to implement thus far is "trust". Although trust is a core value in society, it can be different kinds of trust (trust that you can obtain certain goods, trust in banks and stores, trust that you will be safe when being out in society). Our current interpretation is that trust depends on the other indicators and that is thus might be sufficient to only model them.

\subsection{Replay-ability}

After a short introduction into the learning goals, the computer simulation environment, the start scenario, and the way how the team can choose actions to influence the scenario, the team can play an optional number of rounds. When the start scenario is introduced the simulation is paused at day 1, day 3, day 6 and day 10 to show how the performance measures slowly deteriorate.

When the team later plays itself and chooses actions the simulation-games is initially paused at the same moments to be able to compare the new performance statistics with the earlier ones. Typically, it takes 10 to 20 minutes to discuss and decided on actions, so 1 to $1 \frac{1}{2}$ hour to play the full scenario once. Our expectation is that teams might succeed to play 3 rounds on a half-day (leaving time to sum up and debrief the whole playing sessions) and maybe 6-8 rounds one a full day (where the expectation is that playing speed slowly can be increased when the team plays more rounds as they get familiar with the simulation-game).

\section{GAME DESIGN CHALLENGES}

Most design choices have after some iterations and refinements evolved into more permanent choices where motivation why each respective choice was important gradually became more profound. Three design issues have been particularly challenging and are therefore interesting to highlight as potential areas for future research.

\subsection{Validation}

How to validate an unthinkable crisis escalation scenario? Many of the interactions that are simulated in the computer simulation are based on slightly related incidents and expectations of experts we have interviewed. It is however hard to translate observed effects of poorly related cases or judge the imaginary power of the experts. There might be certain interactions that are hard to imagine and which are not correctly represented in our current simulation. Normally, when building a simulation of an existing system, there is some kind of real data to validate against. As the purpose of crisis scenarios is to be far from the current equilibrium state, it is hard to foresee or imagine what relevant (new) elements and (new) interactions and dependencies are. An interesting future research area is therefore to develop methods and tools to improve the validation of crisis scenarios and simulations.

\subsection{Fidelity and playability}

A major concern in our current design is that players easily can get stuck in details. Multiplying 25 stores and several other actors with 3 decision points in time and roughly 15 different types of actions that each individual store can pick at each point in time results over 1000 potential actions which can be combined in infinite variations. Even though our simulation is a strong simplification of the actual complexity of our society, players might easily get lost here. It has particularly been clear that players easily can zoom in on individual decisions in individual stores and loose the "overall society helicopter view". This is a typical risk of introducing a detailed computer simulation in the role playing simulation.

In our current design discussions different options are explored to handle this issue. One is to develop facilitator strategies to keep the playing teams on track (while keeping the fine granularity of the computer simulation interface). Another is simplifying the computer simulation interface (i.e. limiting the amount or granularity of actions to be taken \& decreasing the number of performance statistics). The latter has the danger that the simulation becomes to abstract and transferability between simulation-game learning and value of the lessons learned in real society is lost.

\subsection{Time per playing round and number of rounds}

A closely related concern is the number of playing rounds and the time per playing round for discussion in the team. More round is preferable, but they should not become so short that players quit discussing their motivations and just guess. On the other hand, teams might get stuck in endless discussions about which actions to choose without ever implementing them in the computer simulation.

Here, well-experienced facilitators are currently seen as the major viable option to fix this challenge. Alternative options could be to allow for playing the simulation independently at a distance after participating in the first facilitated team session. 


\section{DISCUSSION AND CONCLUSION}

During the last two full day workshops where the latest version of the simulation-game was tested it was concluded (by designers and potential players, i.e. representatives from societal sectors) that the current design potentially can increase insight in collective critical infrastructure resilience. The main challenge is to make sure that the team who plays the game does not get stuck in details (due to complexity) and that the game facilitation is of such quality that a reasonable playing speed and number of playing rounds is achieved in a session, while at the same time team players experience to have sufficient time in each playing round to come to thoughtful and well-motivated action packages.

Researchers and practitioners can benefit from an increased insight into the challenges of designing simulation-games for critical infrastructure resilience analysis and training, as documented in this paper. Combining the insights from our design process with insights from alternative applications and approaches can increase the quality of our designs and thereby subsequently improve overall critical infrastructure resilience in society.

\section{ACKNOWLEDGMENTS}

This research was supported by Grant 2016-3046 of the Swedish Civil Contingencies Agency.

\section{REFERENCES}

Alcaraz, C. \& Zeadally, S. 2015. Critical infrastructure protection: Requirements and challenges for the 21st century. International Journal of Critical Infrastructure Protection 8: 53-66.

Ansell, C., Boin, A. \& Keller, A. 2010. Managing Transboundary Crises: Identifying the Building Blocks of an Effective Response System. Journal of Contingencies and Crisis Management 18: 195-207.

Boin, A. \& McConnell, A. 2007. Preparing for critical Infrastructure Breakdowns: The Limits of Crisis Management and the Need of Resilience. Journal of Contingencies and Crisis Management 15(1): 50-59.

Berggren, P., Johansson, B., Baroutsi, N., Turcotte, I. \& Tremblay, S. 2014. Assessing team focused behaviors in emergency response teams using the shared priorities measure. Proceedings of the 11th International ISCRAM Conference, University Park, Pennsylvania, USA: 130-134.

Bergström, J., van Winsen, R \& Henriqson, E. 2015. On the rationale of resilience in the domain of safety: A literature review. Reliability Engineering and System Safety 141: 131-141

Eisenhardt, K.M. \& Graebner, M.E. 2007. Theory Building from Cases: Opportunities and Challenges. Academy of Management Journal 50(1): 25-32.
Hagen, J.M. 2016. Cyber security - The Norwegian way. International Journal of Critical Infrastructure Protection 14: 41-42.

Hevner, A., March, S., Park, J., \& Ram, S. 2004. Design Science in Information Systems Research. MIS Quarterly 28(1): 75-105.

Hollnagel, E. 2013. A tale of two safeties. Nuclear Safety and Simulation 4(1): 1-9.

Johansson, B. \& Lundberg, J. 2010. Engineering Safe Aviation Systems - Balancing Resilience and Stability. In: D.J Garland, J.A Wise \& V.D. Hopkin (Eds.), Handbook of Aviation Human Factors: 6-1 to 6-8. Boca Raton: CRC Press.

Laere, J. van 2003. Coordinating distributed work, Exploring situated coordination with gaming-simulation. Doctoral dissertation, Delft, The Netherlands: Delft University of Technology.

Laere, J. van, Vreede, G.J. de., \& Sol, H.G. 2006. A social simulation game to explore future coordination in knowledge networks at the Amsterdam Police Force. Journal of Production Planning and Control 17(6): 558-568.

Laere, J. van, Berggren, P., Gustavsson, P., Ibrahim, O., Johansson, B., Larsson, A., Lindqwister, T, Olsson, L. \& Wiberg, C. 2017a. Challenges for critical infrastructure resilience: cascading effects of payment system disruptions. Proceedings of the 14th International Conference on Information Systems for Crisis Response and Management Albi, France, 21-24 May 2017 (ISCRAM2017): 281-292.

Laere, J. van, Ibrahim, O., Larsson, A., Olsson, L., Johansson, B., \& Gustavsson P. 2017b. Analyzing the implications of design choices in existing simulationgames for critical infrastructure resilience. Proceedings of the International Simulation and Gaming Association's conference (ISAGA), Delft, The Netherlands, 10-14 July 2017.

Laugé, A., Hernantes, J., and Sarriegi, J.M: 2015. Critical infrastructure dependencies: A holistic, dynamic and quantitative approach. International Journal of Critical Infrastructure Protection 8: 16-23.

Mayer I.S. 2009. The gaming of policy and the politics of gaming: A review. Simulation \& Gaming 40(6): $825-862$.

Meijer S.A. 2009. The organization of transactions: Studying supply networks using gaming simulation. Wageningen, The Netherlands: Wageningen Academic Publishers.

MSB 2009-3309 2010. Gemensamma rutiner, uppdrag inom SOES. (Swedish Civil Contingencies Agency, Shared routines, assignment within Collaboration Area Economic Security), available at: https://www.msb.se/Upload/ Forebyggande/Krisberedskap/Samverkansomraden/ Gemensamma\%20rutiner_ver\%201.0.pdf.

Van Eeten, M., Nieuwenhuis, A., Luijf, E., Klaver, M. \& Cruz, E. 2011. The state and the threat of cascading failure across critical infrastructures: The implications of empirical evidence from media incident reports. Public Administration 89: 381-400.

Weick, K.E. \& Roberts, K. 1993. Collective mind in organizations: Heedful interrelating on flight decks. Administrative science quarterly 38(3): 357-381.

Weick, K.E. \& Sutcliffe, K.M. 2007. Managing the unexpected. Resilient performance in and age of uncertainty. San Francisco: Jossey Bass. 